\title{
27. CLAY MINERALOGY OF SEDIMENTS FROM THE GALICIA MARGIN, ODP LEG 1031
}

\author{
Audrey W. Meyer, Ocean Drilling Program, Texas A\&M University, College Station, Texas \\ and \\ Thomas A. Davies, Institute for Geophysics, University of Texas at Austin, Austin, Texas
}

\begin{abstract}
Samples from Cenozoic and Mesozoic sediments drilled during ODP Leg 103 were analyzed for clay mineralogy. Kaolinite, chlorite, illite, and smectite occur in most of the samples studied; palygorskite and sepiolite occur principally in Paleogene and Eocene brown clays. In general, throughout the section deposited in the syn- and post-rift period, the more calcareous-rich pelagic sediments contain greater amounts of smectite and lesser amounts of kaolinite and chlorite than do the calcareous-poor terrigenous sediments. The clay data presented here also lend support to the general paleoenvironmental interpretation of a relatively warm climate throughout the Cretaceous and early Tertiary and a relatively cooler climate from the Oligocene to the present day.
\end{abstract}

\section{INTRODUCTION}

The Galicia margin, northwest of the Iberian Peninsula, is a sediment-starved passive continental margin (Montadert et al., 1974, 1979a; Groupe Galice, 1979; Boillot et al., 1979, 1980). Tilted rift blocks, trending north and dipping gently east, form a series of half-grabens. On the western, uplifted side of some of these blocks, basement and possibly pre-rift sedimentary rocks crop out, making this an ideal margin to investigate the basement and oldest sedimentary strata by Ocean Drilling Program (ODP) drilling. Principal objectives of coring and logging during Leg 103 included studying the history of rifting, subsidence, and sedimentation on this margin and the relation of these processes to the initiation and progressive opening of the adjacent North Atlantic. These results would complement earlier drilling on the Iberian margin at Site 398, drilled during Deep Sea Drilling Project (DSDP) Leg 47B (Sibuet, Ryan, et al., 1979).

Five sites were drilled during Leg 103 (Fig. 1). Sites 638, 639, and 641 extended the results of Leg 47B by coring through the syn- and pre-rift strata, possibly to the top of basement near the western edge of the margin. Site 637 , located at the western edge of the margin close to the boundary between oceanic and continental crust, recovered presumed upper mantle peridotite. Drilling at Site 640 was on the summit of the ridge closest to the oceanic/continental crust transition, over the deep S reflector widely believed to represent the ductile/brittle boundary within continental crust (Montadert et al., 1979b). Preliminary results from these drill sites are reported in the Leg 103 Initial Report (Boillot, Winterer, et al., 1987); more detailed results from shorebased studies following the cruise are given in the various specialty chapters published in this volume.

The cores recovered at these five sites include a great variety of sedimentary rock types, in addition to a suite of volcanic and metamorphic rocks. The purpose of this study was to document the clay mineralogy of the recovered sediments, particularly in the Cenozoic sections of the drill sites. Results presented in this paper, together with results presented in the Leg 103 site chapters (Boillot, Winterer, et al., 1987), in other specialty chapters in this volume, and in the Leg 47B volume (Sibuet, Ryan, et al., 1979), document temporal and areal changes in sediment sources and modes of sediment deposition on the Galicia margin.

${ }^{1}$ Boillot, G., Winterer, E. L., et al., 1988. Proc. ODP, Sci. Results, 103: College Station, TX (Ocean Drilling Program).

\section{METHODS}

$\mathrm{X}$-ray diffraction (XRD) analyses were made on sediments from all five sites drilled during ODP Leg 103. In general, one or two samples were analyzed from every core, except where core recovery was poor. Attempts were made to sample representative sediment types from each core; where sediment types showed considerable variation, more closely spaced samples were taken.

Samples were prepared and analyzed according to the procedure outlined by Hein et al. (1976). X-ray patterns were run on a Philips ADP $3520 \mathrm{X}$-ray diffractometer with nickel-filtered $\mathrm{Cu}-\mathrm{K}_{\alpha}$ radiation and machine settings of $40 \mathrm{kV}$ and $35 \mathrm{~mA}$. Unglycolated profiles were run from $2^{\circ}-15^{\circ} 2 \theta$; glycolated profiles were run from $2^{\circ}-15^{\circ} 2 \theta$ and $24^{\circ}-26^{\circ} 2 \theta$. Clay minerals were identified by their characteristic basal XRD maxima, or "peaks." Semiquantitative clay percentages were determined on the glycolated X-ray analog records by the peak area method (Milliman and Bornhold, 1973), multiplied by Biscaye's (1965) weighting factors, and normalized to $100 \%$ (Tables 1 and 2). Kaolinite and chlorite are reported together where their percentages could not be differentiated on the $24^{\circ}-26^{\circ} 2 \theta$ glycolated profile.

Table 1 lists both unweighted and weighted peak areas, for ease in later recalculation of the data to compare with other clay mineralogical results generated with different weighting factors. The total area under the sum of the clay peak areas serves as an estimate of the quality of the percentages determined. Percentages were not calculated for profiles with poorly defined peaks or very small peak areas; these samples are omitted from Table 1. Reproducibility of results is $\pm 5 \%$.

\section{RESULTS}

\section{General Comments}

The clay component of sediments recovered during Leg 103 contains kaolinite, chlorite, illite, and smectite, in varying proportions. In a few samples, palygorskite and sepiolite are also present. Characteristic peaks and correction factors are given in Table 2. Semiquantitative percentages of the minerals are plotted vs. depth below seafloor in Figures 2 through 7 . The principal downhole trends in clay mineralogy at each Leg 103 site are discussed below, in site-chronologic order.

\section{Site 637}

Drilling at Site 637 was on the eastern flank of a buried ridge of serpentinized, foliated peridotite (Fig. 1). This peridotite is buried beneath a 212 -m-thick section of sediments, divided into three lithologic units that are described both in the "Site 637" chapter (Boillot, Winterer, et al., 1987) and in Comas and Maldonado (this volume). Figure 2 shows the downhole trends in clay mineralogy at this site. 


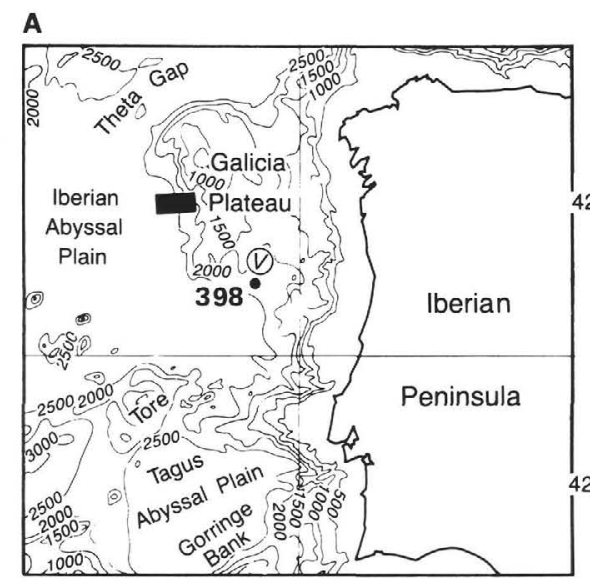

\section{B}
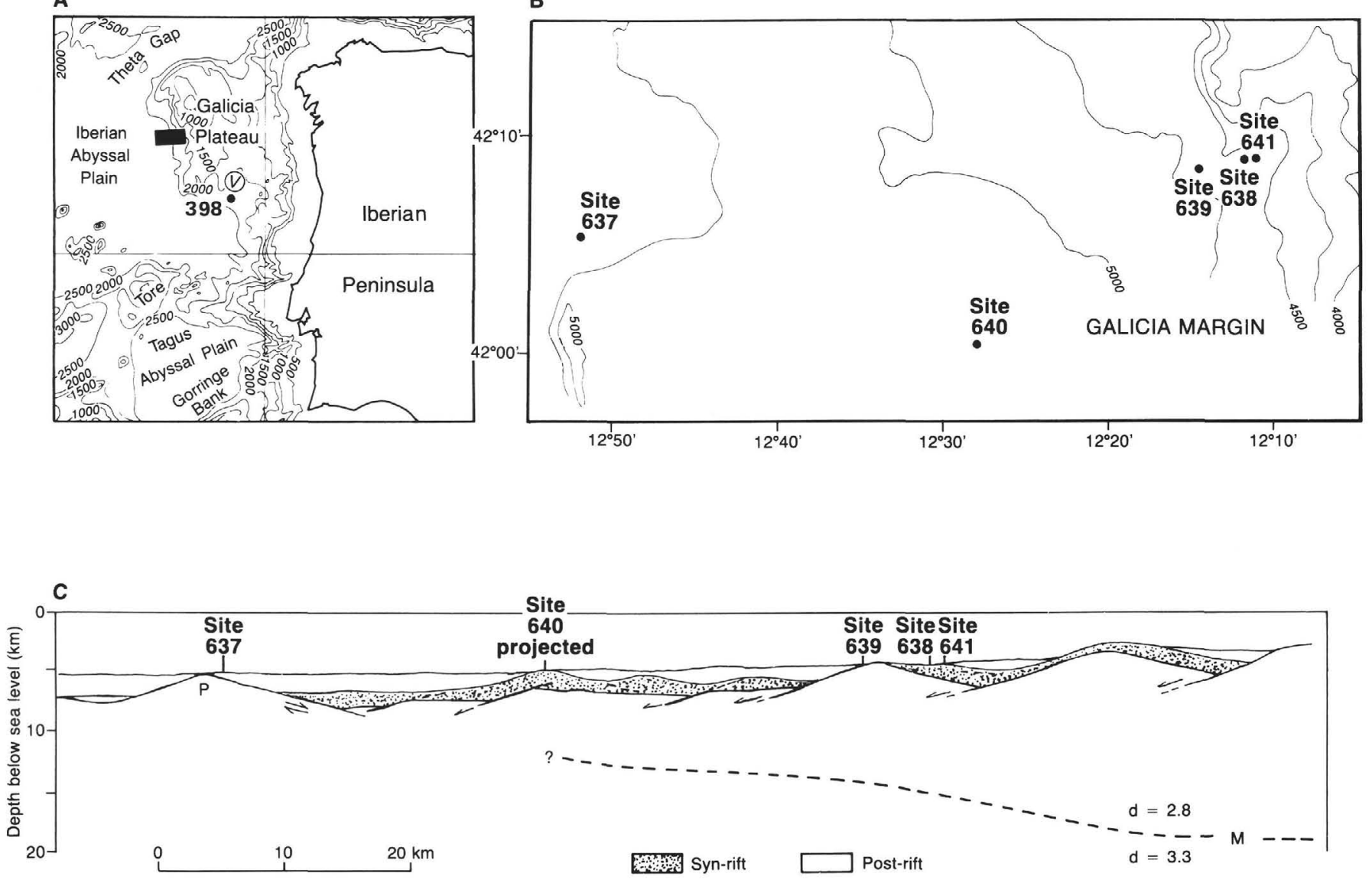

Figure 1. A. Location map of Galicia margin region northwest of Iberian Peninsula. Bathymetric contours in meters. Box indicates area of ODP Leg 103 drilling. Black dot shows location of Site 398 drilled during DSDP Leg 47B south of Vigo Seamount (V). B. Bathymetric map showing location of sites drilled during Leg 103. Modified from Sea Beam map. Contour interval, $250 \mathrm{~m}$. Location of map shown in Figure 1A. C. Schematic cross section across Galicia margin drawn from seismic data and drilling results. $\mathrm{P}=$ peridotite ridge. Low-angle listric faults beneath half-grabens are hypothetical.

Lithologic Unit I (0-135 m below seafloor [mbsf]) consists of upper Pliocene to upper Pleistocene turbidites, interbedded with nannofossil marl. The turbidites average about $25 \mathrm{~cm}$ in thickness. They consist of a graded, gray silt layer, structureless or with parallel laminae, that passes upward into an olive-gray clay. A light gray nannofossil marl commonly overlies the silt-clay couplet and is typically homogenous. The origin of the nannofossil marl layers is not entirely clear, though some show evidence of redeposition (i.e., little dissolution of nannofossils though the water depth of the site is near the carbonate compensation depth (CCD), presence of a small percentage of reworked older species, etc.; see "Site 637" chapter; Boillot, Winterer, et al., 1987). The silt layers become thinner and less abundant downsection.

No clay mineralogy samples were taken from Cores 103-637AIR through 103-637A-5R because of little or no core recovery. Approximately two to three samples were taken from each of the remaining cores in lithologic Unit I (Cores 103-637A-6R through 103-637A-15R), representing the silt and clay turbidite couplets as well as the nannofossil marl. The clay component in these sediments averages about $20 \%$ kaolinite, $15 \%$ chlorite, $50 \%$ illite, and $15 \%$ smectite. The abundance of smectite has the widest range (from $0 \%$ to $29 \%$ ) and is related to the carbonate content: the nannofossil marls typically have more smectite than the clay and silty clay turbidites. The large fluctuations in the clay profile reflect the overall heterogeneity of the sediment types included in this unit.
Lithologic Unit II (135-185 mbsf) consists of upper Miocene to middle Pliocene brown clay and nannofossil marl and includes some distinct slumped intervals. These sediments were interpreted aboard ship as being pelagic sediment and weathering products from the underlying basement, slumped off the basement hill (see "Site 637" chapter; Boillot, Winterer, et al., 1987).

Most of the samples taken from lithologic Unit II consist of nannofossil marl. The clay mineralogy of these marls is similar to that of the marls sampled from lithologic Unit I, averaging $20 \%$ kaolinite, $15 \%$ chlorite, $40 \%$ illite, and $25 \%$ smectite. The silt and clay turbidite layer that was closely sampled in Core 103-637A-17R shows the same increase in amount of smectite upsection (i.e., toward the capping marl) as seen in similar layers in lithologic Unit I.

Lithologic Unit III (185-212 mbsf) consists of reddish brown and grayish brown clay with some exotic lumps of light-colored clay. The brown clays include zeolites, glass, iron oxide particles, and a small percentage of silt-sized terrigenous material (see "Site 637" chapter; Boillot, Winterer, et al., 1987). Based on ichthyoliths, this unit is of Paleogene-Eocene age (Doyle, this volume; Moullade et al., this volume). It was interpreted aboard ship as being a mixture of slowly accumulating pelagic clay, weathering products from the underlying basement, and continental detritus brought to the site by dilute nepheloid or turbidity currents. 
The clay mineralogy of the reddish brown and grayish brown clays of lithologic Unit III is distinctly different than that of the overlying units. Only very small amounts of kaolinite and chlorite $(2 \%-5 \%)$ and smectite $(7 \%-13 \%)$ are present. Illite typically comprises $12 \%-13 \%$ of the clay component, though the uppermost sample contains $36 \%$. The bulk of the clay component consists of palygorskite $(29 \%-46 \%)$ and sepiolite $(36 \%$ $48 \%$ ). The significance of this distinctive clay assemblage is discussed in the following text.

\section{Site 638}

Site 638 is on a rift block close to the western edge of the Galicia margin (Fig. 1). Together with Site 639, these sites were drilled to yield data on the very early history of the margin, on the timing of rifting, and on the timing of paleoenvironmental changes in this region of the North Atlantic. A total of $547.2 \mathrm{~m}$ was penetrated in Holes $638 \mathrm{~B}$ and $638 \mathrm{C}$ at this site, recovering post- and syn-rift sediments that are divided into three lithologic units. Clay mineral analyses were run on samples from each of these units; the data are presented in Table 1 and graphed in Figure 3.

Lithologic Unit I (0-183.6 mbsf), recovered only in Hole $638 \mathrm{~B}$, consists primarily of upper Miocene to Pleistocene white, pale gray, and pale greenish gray clayey nannofossil ooze and chalk. Carbonate content increases downhole. The sediments are generally very soft and have been deformed by drilling so that original sedimentary structures are not generally observable. These carbonates are interpreted as a pelagic submarine valley-fill deposited above the CCD. The clay component of samples analyzed from this unit averages about $20 \%$ kaolinite, $15 \%$ chlorite, $45 \%$ illite, and $20 \%$ smectite. However, the clay profiles for samples collected from Cores 103-638B-7R through $103-638 \mathrm{~B}-12 \mathrm{R}$ are poor, making interpretation of clay mineral percentages tenuous in this section.

Included within the carbonates of lithologic Unit I are two discrete layers of firm clay with less than $5 \%$ carbonate (Samples 103-638B-10R-2, 90-105 cm, and 103-638B-18R-1, 0-65 cm). The former layer is green with black, zeolite-rich laminae; the latter is reddish brown. Both layers include a jumble of clasts that have the appearance of debris flows; they are certainly redeposited because they contain Cretaceous microfossils (see "Site 638" chapter; Boillot, Winterer, et al., 1987). The reddish brown clay layer in Core 103-638B-18R contains about $0 \%-20 \%$ kaolinite and chlorite, $30 \%$ illite, $30 \%$ smectite, and 15\%-35\% palygorskite. The clay layer in Core 103-638B-10R was not sampled.

Lithologic Unit II (183.6-298.4 mbsf) is divided into two subunits. The upper subunit (Subunit IIA) consists of upper Barremian bioturbated and weakly laminated micritic limestone and marlstone, interbedded with 1 - to 5-cm-thick couplets of silty claystone and marlstone rich in terrigenous material. The former are interpreted as pelagic sediments deposited above the $\mathrm{CCD}$, and the couplets are probably turbidites, resembling $\mathrm{T}_{d}-\mathrm{T}_{e}$ beds of the Bouma sequence. The lower subunit (Subunit IIB) consists of alternations of Valanginian-Hauterivian bioturbated nannofossil marlstone and more clay-rich nannofossil marlstone, with many slumped intervals. Of the relatively few samples taken for clay analyses from Unit II, most are from calcareous claystone and marlstone sediment types. Clay mineralogies vary considerably, but average about $20 \%$ kaolinite + chlorite, $40 \%$ illite, and $40 \%$ smectite. Kaolinite and chlorite could not be differentiated on many of the profiles but, where possible, they appear to be present in roughly equal amounts.

Lithologic Unit III (298.4-547.2 mbsf) is distinguished by the occurrence of abundant terrigenous turbidites and is of Valanginian age. It is divided into two subunits. The upper subunit (Subunit IIIA) consists of silty claystone and nannofossil marl- stone couplets, with several layers of sandstone turbidites up to $10 \mathrm{~cm}$ thick. The lower subunit (Subunit IIIB) is characterized by thick beds of terrigenous medium- to coarse-grained arkosic sandstone, interbedded with minor siltstone/claystone and marlstone (for detailed petrographic descriptions of the sandstone layers see Johnson, this volume; Winterer et al., this volume). The sandstone and siltstone/claystone layers are interpreted as turbidites and the marlstone as pelagic material deposited during the time intervals between turbidity current events. Clay samples from this unit were collected primarily from the claystone intervals. The clay mineralogy of these samples averages $20 \%$ kaolinite, $20 \%$ chlorite, $45 \%$ illite, and $15 \%$ smectite.

\section{Site 639}

Site 639 was situated close to Site 638 (Fig. 1) in order to penetrate through the syn-rift sequence into the pre-rift carbonate platform sequence and crystalline basement-an objective we failed to achieve at Site 638 because of hole instability. A total of six holes was drilled at Site 639; the operational reasons behind this are in the "Site 639" chapter (Boillot, Winterer, et al., 1987). By piecing together the sections at these holes, the resulting generalized stratigraphic section can be divided into six lithologic units. These lithologic units are described only briefly here (see "Site 639" chapter for further details; Boillot, Winterer, et al., 1987). Few samples were taken for clay mineralogic analyses at this site, largely because of the poor core recovery.

Lithologic Unit I consists of Neogene clayey nannofossil ooze, nannofossil marl, and calcareous clay, occurring as vague, rhythmic alternations on a $10-\mathrm{cm}$ to $2.5-\mathrm{m}$ scale. These sediments are interpreted as pelagic carbonates probably deposited above the CCD. The more clay-rich intervals may represent increased influx of terrigenous material during Pliocene-Pleistocene glaciations, perhaps as distal turbidity flows or as nepheloid layers. Lithologic Unit I was cored in four of the six drill holes and was sampled for clay mineralogy analyses at three of these holes (Holes 639A, 639B, and 639D; see Fig. 4). The clay component of these sediments in the three holes averages about $10 \%$ kaolinite, $10 \%$ chlorite, $45 \%$ illite, and $35 \%$ smectite.

Lithologic Unit II consists of dark brown to light reddish brown clay, with clayey silt and limonite-stained sandy patches. It was recovered only in Hole 639C, and is dated as PaleoceneEocene age by ichthyoliths (Doyle, this volume). A total of four samples from this unit were analyzed for clay mineralogy. These samples contain an average of about $30 \%$ illite, $20 \%$ smectite, $30 \%$ palygorskite, and smaller amounts of kaolinite $(13 \%)$ and chlorite $(7 \%)$.

Lithologic Unit III, recovered only in Hole 639A, consists of lower Valanginian, mottled, pale yellow, calpionellid-bearing marl and marlstone. Thin sandstone interbeds and clasts were probably deposited by low-density turbidity flows during what was otherwise a period of predominantly pelagic sedimentation. The clay component of samples analyzed from this unit consists of about $20 \%$ chlorite, $35 \%$ smectite, $45 \%$ illite, and little or no kaolinite (Fig. 4).

Lithologic Unit IV consists of dolomite, commonly fractured and vuggy, with internal sediments in fractures and vugs. Its age is uncertain, and no samples were taken for clay analyses.

Lithologic Unit V consists of Tithonian limestone, clayey limestone, marlstone, and sandstone. Only two samples were analyzed for clay mineralogy. Sample 103-639D-7R-2, 80-82 cm, a calcareous clay, consists of $9 \%$ kaolinite + chlorite, $13 \%$ smectite, and 78\% illite. Sample 103-639D-8R-2, 14-16 cm, a siltstone, contains $5 \%$ kaolinite + chlorite, $62 \%$ smectite, and $33 \%$ illite. In both instances, kaolinite and chlorite could not be differentiated because of the overall minor quantities of those clay minerals in the samples. 
Table 1. Leg 103 clay mineralogy data.

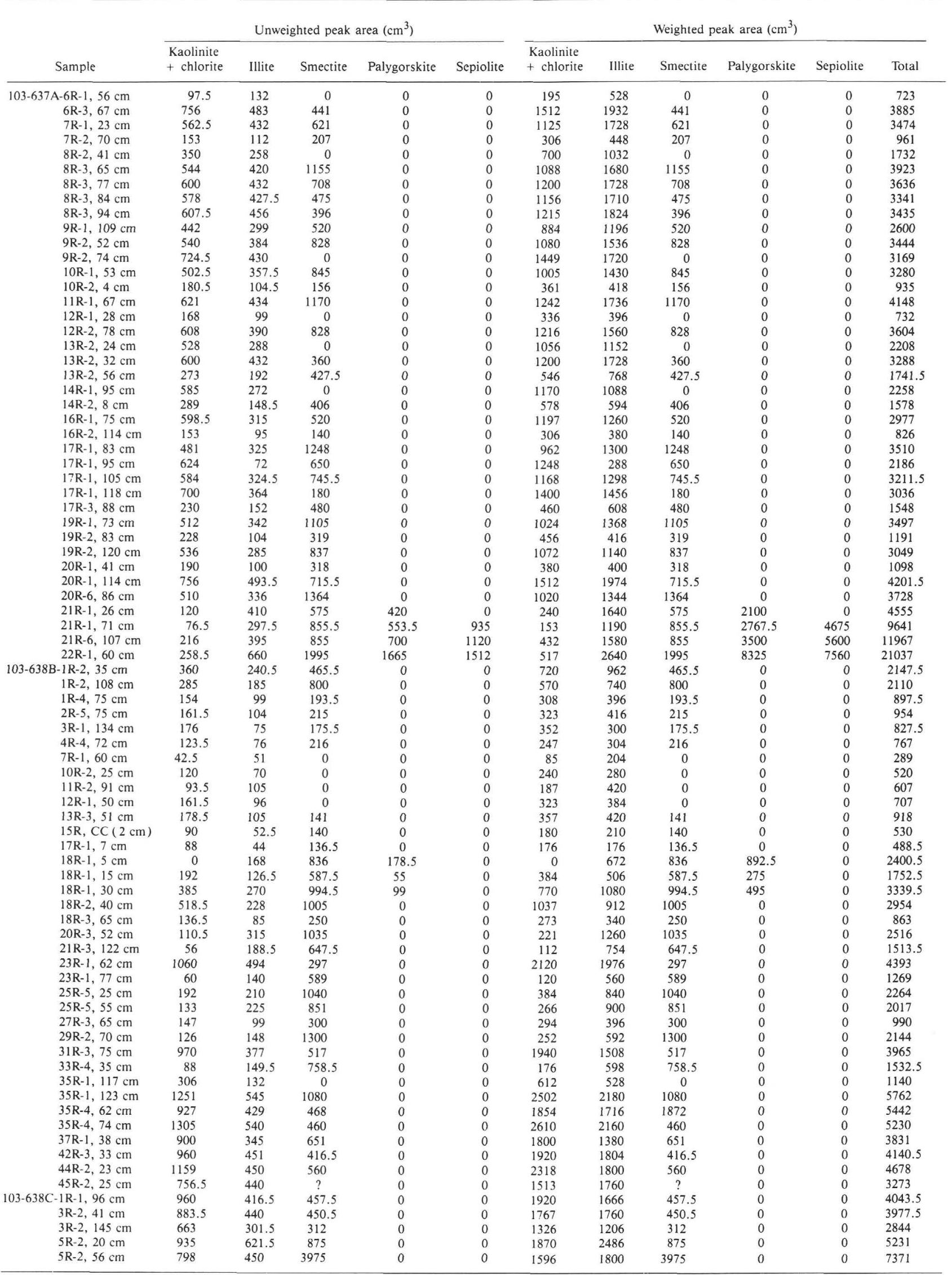


Table 1 (continued).

\begin{tabular}{|c|c|c|c|c|c|c|c|c|}
\hline \multicolumn{5}{|c|}{ Clay mineral percentage } & \multicolumn{2}{|c|}{ Peak height $(\mathrm{cm})$} & \multicolumn{2}{|c|}{$\begin{array}{l}\text { Clay mineral } \\
\text { percentage }\end{array}$} \\
\hline $\begin{array}{l}\text { Kaolinite } \\
+ \text { chlorite }\end{array}$ & Illite & Smectite & Palygorskite & Sepiolite & Kaolinite & Chlorite & Kaolinite & Chlorite \\
\hline 27 & 73 & 0 & 0 & 0 & $?$ & $?$ & $?$ & $?$ \\
\hline 39 & 50 & 11 & 0 & 0 & 77 & 49 & 24 & 15 \\
\hline 32 & 50 & 18 & 0 & 0 & 71 & 47 & 19 & 13 \\
\hline 32 & 47 & 21 & 0 & 0 & $?$ & ? & $?$ & $?$ \\
\hline 40 & 60 & 0 & 0 & 0 & 50 & 35 & 24 & 16 \\
\hline 28 & 43 & 29 & 0 & 0 & 59 & 43 & 16 & 12 \\
\hline 33 & 48 & 19 & 0 & 0 & $?$ & $?$ & $?$ & $?$ \\
\hline 35 & 51 & 14 & 0 & 0 & 65 & 49 & 20 & 15 \\
\hline 35 & 53 & 12 & 0 & 0 & 75 & 42 & 22 & 13 \\
\hline 34 & 46 & 20 & 0 & 0 & 50 & 38 & 19 & 15 \\
\hline 31 & 45 & 24 & 0 & 0 & 74 & 42 & 20 & 11 \\
\hline 46 & 54 & 0 & 0 & 0 & $?$ & $?$ & $?$ & $?$ \\
\hline 30 & 44 & 26 & 0 & 0 & 70 & 49 & 18 & 12 \\
\hline 38 & 45 & 17 & 0 & 0 & 18 & 13 & 22 & 16 \\
\hline 30 & 42 & 28 & 0 & 0 & 62 & 46 & 17 & 13 \\
\hline 46 & 54 & 0 & 0 & 0 & 24 & 17 & 27 & 19 \\
\hline 34 & 43 & 23 & 0 & 0 & 74 & 55 & 20 & 14 \\
\hline 48 & 52 & 0 & 0 & 0 & 68 & 42 & 30 & 18 \\
\hline 36 & 53 & 11 & 0 & 0 & 73 & 46 & 22 & 14 \\
\hline 31 & 44 & 25 & 0 & 0 & 35 & 20 & 20 & 11 \\
\hline 52 & 48 & 0 & 0 & 0 & 101 & 59 & 33 & 19 \\
\hline 36 & 38 & 26 & 0 & 0 & 33 & 20 & 22 & 14 \\
\hline 40 & 42 & 18 & 0 & 0 & $?$ & $?$ & $?$ & $?$ \\
\hline 37 & 46 & 17 & 0 & 0 & ? & $?$ & ? & $?$ \\
\hline 27 & 37 & 36 & 0 & 0 & 75 & 40 & 18 & 9 \\
\hline 57 & 13 & 30 & 0 & 0 & 71 & 44 & 35 & 22 \\
\hline 36 & 41 & 23 & 0 & 0 & 76 & 47 & 22 & 14 \\
\hline 46 & 48 & 6 & 0 & 0 & $?$ & ? & $?$ & $?$ \\
\hline 30 & 39 & 31 & 0 & 0 & 22 & 12 & 19 & 11 \\
\hline 29 & 39 & 32 & 0 & 0 & 60 & 35 & 18 & 11 \\
\hline 38 & 35 & 27 & 0 & 0 & 24 & 14 & 24 & 14 \\
\hline 35 & 37 & 28 & 0 & 0 & 68 & 44 & 21 & 14 \\
\hline 35 & 36 & 29 & 0 & 0 & 21 & 17 & 19 & 16 \\
\hline 36 & 47 & 17 & 0 & 0 & $?$ & $?$ & $?$ & $?$ \\
\hline 27 & 36 & 37 & 0 & 0 & 57 & 35 & 17 & 10 \\
\hline 5 & 36 & 13 & 46 & 0 & 17 & 22 & 2 & 3 \\
\hline 2 & 12 & 9 & 29 & 48 & 25 & 29 & 1 & 1 \\
\hline 4 & 13 & 7 & 29 & 47 & 24 & 31 & 2 & 2 \\
\hline 2 & 13 & 9 & 40 & 36 & 39 & 27 & 1 & 1 \\
\hline 33 & 45 & 22 & 0 & 0 & 44 & 34 & 19 & 14 \\
\hline 27 & 35 & 38 & 0 & 0 & 37 & 28 & 15 & 12 \\
\hline 34 & 44 & 22 & 0 & 0 & 14 & 11 & 19 & 15 \\
\hline 34 & 44 & 22 & 0 & 0 & 17 & 12 & 20 & 14 \\
\hline 43 & 36 & 21 & 0 & 0 & $?$ & $?$ & $?$ & $?$ \\
\hline 32 & 40 & 28 & 0 & 0 & 13 & 10 & 18 & 14 \\
\hline 29 & 71 & 0 & 0 & 0 & 9 & 10 & 14 & 15 \\
\hline 46 & 54 & 0 & 0 & 0 & $?$ & $?$ & $?$ & $?$ \\
\hline 31 & 69 & 0 & 0 & 0 & $?$ & $?$ & $?$ & $?$ \\
\hline 46 & 54 & 0 & 0 & 0 & 15 & 12 & 26 & 20 \\
\hline 39 & 46 & 15 & 0 & 0 & 18 & 10 & 25 & 14 \\
\hline 34 & 40 & 26 & 0 & 0 & 13 & 9 & 20 & 14 \\
\hline 36 & 36 & 28 & 0 & 0 & 13 & 7 & 23 & 13 \\
\hline 0 & 28 & 35 & 37 & 0 & 0 & 0 & 0 & 0 \\
\hline 22 & 29 & 34 & 15 & 0 & 32 & 18 & 14 & 8 \\
\hline 23 & 32 & 30 & 15 & 0 & 58 & 20 & 17 & 6 \\
\hline 35 & 31 & 34 & 0 & 0 & 62 & 43 & 21 & 14 \\
\hline 32 & 39 & 29 & 0 & 0 & $?$ & $?$ & $?$ & $?$ \\
\hline 9 & 50 & 41 & 0 & 0 & $?$ & $?$ & ? & $?$ \\
\hline 7 & 50 & 43 & 0 & 0 & $?$ & $?$ & $?$ & $?$ \\
\hline 48 & 45 & 7 & 0 & 0 & $?$ & $?$ & $?$ & $?$ \\
\hline 10 & 44 & 46 & 0 & 0 & $?$ & $?$ & $?$ & $?$ \\
\hline 17 & 37 & 46 & 0 & 0 & 19 & 21 & 8 & 9 \\
\hline 13 & 45 & 42 & 0 & 0 & 14 & 15 & 6 & 7 \\
\hline 30 & 40 & 30 & 0 & 0 & 8 & 8 & 15 & 15 \\
\hline 12 & 27 & 61 & 0 & 0 & 22 & 10 & 8 & 4 \\
\hline 49 & 38 & 13 & 0 & 0 & $?$ & $?$ & $?$ & $?$ \\
\hline 11 & 39 & 50 & 0 & 0 & ? & $?$ & $?$ & ? \\
\hline 54 & 46 & 0 & 0 & 0 & 29 & 28 & 27 & 27 \\
\hline 43 & 38 & 19 & 0 & 0 & 83 & 86 & 21 & 22 \\
\hline 34 & 32 & 34 & 0 & 0 & 90 & 93 & 17 & 17 \\
\hline 50 & 41 & 9 & 0 & 0 & 74 & 55 & 28 & 22 \\
\hline 47 & 36 & 17 & 0 & 0 & 89 & 90 & 23 & 24 \\
\hline 46 & 44 & 10 & 0 & 0 & 86 & 84 & 23 & 23 \\
\hline 50 & 38 & 12 & 0 & 0 & 84 & 86 & 25 & 25 \\
\hline 46 & 54 & $?$ & 0 & 0 & 84 & 84 & 23 & 23 \\
\hline 48 & 41 & 11 & 0 & 0 & 127 & 137 & 23 & 25 \\
\hline 45 & 44 & 11 & 0 & 0 & 94 & 86 & 23 & 22 \\
\hline 47 & 42 & 11 & 0 & 0 & 86 & 81 & 24 & 23 \\
\hline 36 & 47 & 17 & 0 & 0 & 105 & 97 & 19 & 17 \\
\hline 22 & 23 & 54 & 0 & 0 & 112 & 109 & 11 & 11 \\
\hline
\end{tabular}


Table 1 (continued).

\begin{tabular}{|c|c|c|c|c|c|c|c|c|c|c|c|}
\hline \multirow[b]{2}{*}{ Sample } & \multicolumn{5}{|c|}{ Unweighted peak area $\left(\mathrm{cm}^{3}\right)$} & \multicolumn{6}{|c|}{ Weighted peak area $\left(\mathrm{cm}^{3}\right)$} \\
\hline & $\begin{array}{l}\text { Kaolinite } \\
+ \text { chlorite }\end{array}$ & Illite & Smectite & Palygorskite & Sepiolite & $\begin{array}{l}\text { Kaolinite } \\
+ \text { chlorite }\end{array}$ & Illite & Smectite & Palygorskite & Sepiolite & Total \\
\hline $103-638 \mathrm{C}-8 \mathrm{R}-1,116 \mathrm{~cm}$ & 1270.5 & 495 & 4292 & 0 & 0 & 2541 & 1980 & 4292 & 0 & 0 & 8813 \\
\hline $8 \mathrm{R}-1,125 \mathrm{~cm}$ & 1190 & 700 & 540 & 0 & 0 & 2380 & 2800 & 540 & 0 & 0 & 5720 \\
\hline $9 \mathrm{R}-2,16 \mathrm{~cm}$ & 1208 & 822 & 973.5 & 0 & 0 & 2416 & 3288 & 973.5 & 0 & 0 & 6677.5 \\
\hline $12 \mathrm{R}-1,65 \mathrm{~cm}$ & 756.5 & 498 & 235 & 0 & 0 & 1513 & 1992 & 235 & 0 & 0 & 3740 \\
\hline $14 \mathrm{R}-1,68 \mathrm{~cm}$ & 1521 & 696 & 569.5 & 0 & 0 & 3042 & 2784 & 569.5 & 0 & 0 & 6395.5 \\
\hline $14 \mathrm{R}-2,49 \mathrm{~cm}$ & 1521 & 1147.5 & 904.5 & 0 & 0 & 3042 & 4590 & 904.5 & 0 & 0 & 8536.5 \\
\hline 103-639A-1R-1, $118 \mathrm{~cm}$ & 232.5 & 217 & 651 & 0 & 0 & 465 & 868 & 651 & 0 & 0 & 1984 \\
\hline $2 \mathrm{R}-5,40 \mathrm{~cm}$ & 72 & 112.5 & 532.5 & 0 & 0 & 144 & 450 & 532.5 & 0 & 0 & 1126.5 \\
\hline $3 \mathrm{R}-1,22 \mathrm{~cm}$ & 210 & 240 & 586.5 & 0 & 0 & 420 & 960 & 586.5 & 0 & 0 & 1966.5 \\
\hline $3 \mathrm{R}-4,57 \mathrm{~cm}$ & 154 & 132 & 632.5 & 0 & 0 & 308 & 528 & 632.5 & 0 & 0 & 1468.5 \\
\hline $4 \mathrm{R}-1,20 \mathrm{~cm}$ & 851.5 & 627 & 2146.5 & 0 & 0 & 1703 & 627 & 2146.5 & 0 & 0 & 4476.5 \\
\hline $4 \mathrm{R}-1,29 \mathrm{~cm}$ & 648 & 350 & 759 & 0 & 0 & 1296 & 1400 & 759 & 0 & 0 & 3455 \\
\hline $5 \mathrm{R}-1,54 \mathrm{~cm}$ & 455 & 296 & 1232 & 0 & 0 & 910 & 1184 & 1232 & 0 & 0 & 3326 \\
\hline $6 \mathrm{R}-1,79 \mathrm{~cm}$ & 243 & 265 & 585 & 0 & 0 & 486 & 1060 & 585 & 0 & 0 & 2131 \\
\hline $6 \mathrm{R}-2,100 \mathrm{~cm}$ & 235 & 325 & 1014 & 0 & 0 & 470 & 1300 & 1014 & 0 & 0 & 2784 \\
\hline $7 \mathrm{R}-2,15 \mathrm{~cm}$ & 188 & 228 & 712.5 & 0 & 0 & 376 & 912 & 712.5 & 0 & 0 & 2000.5 \\
\hline $8 \mathrm{R}-1,73 \mathrm{~cm}$ & 175.5 & 176 & 562.5 & 0 & 0 & 351 & 704 & 562.5 & 0 & 0 & 1617.5 \\
\hline $8 \mathrm{R}-2,64 \mathrm{~cm}$ & 275 & 390 & 899 & 0 & 0 & 550 & 1560 & 899 & 0 & 0 & 3009 \\
\hline 103-639B-1R-5, $46 \mathrm{~cm}$ & 165 & 150 & 273 & 0 & 0 & 330 & 600 & 273 & 0 & 0 & 1203 \\
\hline $103-639 \mathrm{C}-2 \mathrm{R}-1,36 \mathrm{~cm}$ & 721 & 575 & 2436 & 221 & 0 & 1442 & 2300 & 2436 & 1105 & 0 & 7283 \\
\hline $2 \mathrm{R}-1,61 \mathrm{~cm}$ & 1117.5 & 851.5 & 1245.5 & 176 & 0 & 2235 & 1703 & 1245.5 & 880 & 0 & 6063.5 \\
\hline $2 \mathrm{R}-1,104 \mathrm{~cm}$ & 186 & 322 & 1085 & 600 & 0 & 372 & 1288 & 1085 & 3000 & 0 & 5745 \\
\hline $2 \mathrm{R}-2,17 \mathrm{~cm}$ & 822 & 948 & 1533 & 550 & 0 & 1644 & 3792 & 1533 & 2750 & 0 & 9719 \\
\hline 103-639D-1W-1, $43 \mathrm{~cm}$ & 352 & 380 & 938 & 0 & 0 & 704 & 1520 & 938 & 0 & 0 & 3162 \\
\hline $7 \mathrm{R}-2,82 \mathrm{~cm}$ & 266.5 & 1200 & 837 & 0 & 0 & 533 & 4800 & 837 & 0 & 0 & 6170 \\
\hline $8 \mathrm{R}-1,14 \mathrm{~cm}$ & 120 & 378 & 2821.5 & 0 & 0 & 240 & 1512 & 2821.5 & 0 & 0 & 4573.5 \\
\hline $103-640 \mathrm{~A}-1 \mathrm{R}-1,51 \mathrm{~cm}$ & 162.5 & 185 & 1537 & 180 & 0 & 325 & 740 & 1537 & 900 & 0 & 3502 \\
\hline $1 \mathrm{R}-1,95 \mathrm{~cm}$ & 280 & 200 & 1275 & 204 & 0 & 560 & 800 & 1275 & 1020 & 0 & 3655 \\
\hline $1 \mathrm{R}-1,118 \mathrm{~cm}$ & 420 & 588 & 2915 & 1175 & 0 & 840 & 2352 & 2915 & 5875 & 0 & 11982 \\
\hline $2 \mathrm{R}-1,72 \mathrm{~cm}$ & 658 & 360 & 1675 & 495 & 0 & 1316 & 1440 & 1675 & 2475 & 0 & 6906 \\
\hline $2 \mathrm{R}-1,128 \mathrm{~cm}$ & 279.5 & 282 & 1091.5 & 637 & 0 & 559 & 1128 & 1091.5 & 3185 & 0 & 5963.5 \\
\hline $2 \mathrm{R}-2,25 \mathrm{~cm}$ & 585 & 473 & 2146.5 & 587.5 & 0 & 1170 & 1892 & 2146.5 & 2937.5 & 0 & 8146 \\
\hline $2 \mathrm{R}-2,37 \mathrm{~cm}$ & 12 & 221 & 2400 & 216 & 0 & 24 & 884 & 2400 & 1080 & 0 & 4388 \\
\hline $2 \mathrm{R}-2,49 \mathrm{~cm}$ & 66 & 493.5 & 2914 & 0 & 0 & 132 & 1974 & 2914 & 0 & 0 & 5020 \\
\hline $3 \mathrm{R}-2,59 \mathrm{~cm}$ & 60 & 180 & 559 & 0 & 0 & 120 & 720 & 559 & 0 & 0 & 1399 \\
\hline $4 \mathrm{R}-1,60 \mathrm{~cm}$ & 123.5 & 186 & 924 & 0 & 0 & 247 & 744 & 924 & 0 & 0 & 1915 \\
\hline $6 \mathrm{R}-1,46 \mathrm{~cm}$ & 620 & 455 & 2131.5 & 0 & 0 & 1240 & 1820 & 2131.5 & 0 & 0 & 5191.5 \\
\hline $6 \mathrm{R}-1,85 \mathrm{~cm}$ & 238 & 297 & 1425 & 0 & 0 & 476 & 1188 & 1425 & 0 & 0 & 3089 \\
\hline $7 \mathrm{R}-1,64 \mathrm{~cm}$ & 603.5 & 583 & 2843.5 & 0 & 0 & 1207 & 2332 & 2843.5 & 0 & 0 & 6382.5 \\
\hline $8 \mathrm{R}-1,37 \mathrm{~cm}$ & 810 & 564 & 1617 & 0 & 0 & 1620 & 2256 & 1617 & 0 & 0 & 5493 \\
\hline $103-641 \mathrm{~A}-1 \mathrm{X}-2,26 \mathrm{~cm}$ & 675 & 636 & 3551 & 0 & 0 & 1350 & 2544 & 3551 & 0 & 0 & 7445 \\
\hline $1 \mathrm{X}-3,56 \mathrm{~cm}$ & 480 & 461.5 & 1736 & 0 & 0 & 960 & 1846 & 1736 & 0 & 0 & 4542 \\
\hline $3 \mathrm{X}-2,135 \mathrm{~cm}$ & 368 & 188 & 984 & 0 & 0 & 736 & 752 & 984 & 0 & 0 & 2472 \\
\hline $4 \mathrm{X}-1,44 \mathrm{~cm}$ & 345 & 203.5 & 897 & 0 & 0 & 690 & 814 & 897 & 0 & 0 & 2401 \\
\hline $5 \mathrm{X}-1,62 \mathrm{~cm}$ & 196 & 112.5 & 1788.5 & 0 & 0 & 392 & 450 & 1788.5 & 0 & 0 & 2630.5 \\
\hline $5 \mathrm{X}-2,95 \mathrm{~cm}$ & 525 & 269.5 & 2660 & 0 & 0 & 1050 & 1078 & 2660 & 0 & 0 & 4788 \\
\hline $6 \mathrm{X}-3,50 \mathrm{~cm}$ & 456 & 266 & 2420.5 & 0 & 0 & 912 & 1064 & 2420.5 & 0 & 0 & 4396.5 \\
\hline $6 \mathrm{X}-5,62 \mathrm{~cm}$ & 378 & 174 & 1192.5 & 0 & 0 & 756 & 696 & 1192.5 & 0 & 0 & 2644.5 \\
\hline $6 \mathrm{X}-7,5 \mathrm{~cm}$ & 560 & 282 & 2835 & 0 & 0 & 1120 & 1128 & 2835 & 0 & 0 & 5083 \\
\hline $103-641 \mathrm{C}-1 \mathrm{R}-1,142 \mathrm{~cm}$ & 0 & 96 & 774 & 149.5 & 0 & 0 & 384 & 774 & 747.5 & 0 & 1905.5 \\
\hline $2 \mathrm{R}-4,22 \mathrm{~cm}$ & 67.5 & 294 & 1920 & 168 & 0 & 135 & 1176 & 1920 & 840 & 0 & 4071 \\
\hline $2 \mathrm{R}-5,46 \mathrm{~cm}$ & 81 & 304 & 1564 & 75 & 0 & 162 & 1216 & 1564 & 375 & 0 & 3317 \\
\hline $3 \mathrm{R}-2,39 \mathrm{~cm}$ & 108 & 370 & 717.5 & 0 & 0 & 216 & 1480 & 717.5 & 0 & 0 & 2413.5 \\
\hline $3 \mathrm{R}-3,9 \mathrm{~cm}$ & 65 & 323 & 2218.5 & 0 & 0 & 130 & 1292 & 2218.5 & 0 & 0 & 3640.5 \\
\hline $4 \mathrm{R}-2,57 \mathrm{~cm}$ & 49.5 & 301 & 2266 & 0 & 0 & 99 & 1204 & 2266 & 0 & 0 & 3569 \\
\hline $4 \mathrm{R}-2,60 \mathrm{~cm}$ & 121 & 595 & 3822 & 0 & 0 & 242 & 2380 & 3822 & 0 & 0 & 6444 \\
\hline $4 \mathrm{R}-2,63 \mathrm{~cm}$ & 93.5 & 595 & 4303.5 & 0 & 0 & 187 & 2380 & 4303.5 & 0 & 0 & 6870.5 \\
\hline $4 \mathrm{R}-4,64 \mathrm{~cm}$ & 78 & 456 & 2322 & 0 & 0 & 156 & 1824 & 2322 & 0 & 0 & 4302 \\
\hline $6 \mathrm{R}-1,12 \mathrm{~cm}$ & 80 & 234 & 1584 & 0 & 0 & 160 & 936 & 1584 & 0 & 0 & 2680 \\
\hline $6 \mathrm{R}-2,52 \mathrm{~cm}$ & 54 & 228 & 2777.5 & 0 & 0 & 108 & 912 & 2777.5 & 0 & 0 & 3797.5 \\
\hline $6 \mathrm{R}-2,60 \mathrm{~cm}$ & 82.5 & 440 & 1815 & 0 & 0 & 165 & 1760 & 1815 & 0 & 0 & 3740 \\
\hline $6 \mathrm{R}-2,88 \mathrm{~cm}$ & 28 & 210 & 2134 & 0 & 0 & 56 & 840 & 2134 & 0 & 0 & 3030 \\
\hline $6 \mathrm{R}-3,78 \mathrm{~cm}$ & 65 & 514.5 & 3473 & 0 & 0 & 130 & 514.5 & 3473 & 0 & 0 & 4117.5 \\
\hline $6 \mathrm{R}-3,80 \mathrm{~cm}$ & 24 & 175 & 1802 & 0 & 0 & 48 & 700 & 1802 & 0 & 0 & 2550 \\
\hline $7 \mathrm{R}-1,51 \mathrm{~cm}$ & 82.5 & 372 & 1209 & 0 & 0 & 165 & 1488 & 1209 & 0 & 0 & 2862 \\
\hline $8 \mathrm{R}-3,51 \mathrm{~cm}$ & 165 & 297 & 1934.5 & 0 & 0 & 330 & 1188 & 1934.5 & 0 & 0 & 3452.5 \\
\hline $9 \mathrm{R}-1,112 \mathrm{~cm}$ & 120 & 422.5 & 1903.5 & 0 & 0 & 240 & 1690 & 1903.5 & 0 & 0 & 3833.5 \\
\hline $9 \mathrm{R}-2,112 \mathrm{~cm}$ & 85 & 370.5 & 1955 & 0 & 0 & 170 & 1482 & 1955 & 0 & 0 & 3607 \\
\hline $10 \mathrm{R}-3,104 \mathrm{~cm}$ & 0 & 165 & 779 & 0 & 0 & 0 & 660 & 779 & 0 & 0 & 1439 \\
\hline $11 \mathrm{R}-1,81 \mathrm{~cm}$ & 78 & 378 & 1200 & 0 & 0 & 156 & 1512 & 1200 & 0 & 0 & 2868 \\
\hline $11 \mathrm{R}-3,54 \mathrm{~cm}$ & 59.5 & 160 & 304.5 & 0 & 0 & 119 & 640 & 304.5 & 0 & 0 & 1063.5 \\
\hline $12 \mathrm{R}-2,97 \mathrm{~cm}$ & 52 & 126 & 340 & 0 & 0 & 104 & 504 & 340 & 0 & 0 & 948 \\
\hline $12 \mathrm{R}-3,50 \mathrm{~cm}$ & 49 & 379.5 & 1260 & 0 & 0 & 98 & 1518 & 1260 & 0 & 0 & 2876 \\
\hline $13 \mathrm{R}-2,111 \mathrm{~cm}$ & 60 & 240 & 1350 & 0 & 0 & 120 & 960 & 1350 & 0 & 0 & 2430 \\
\hline $13 \mathrm{R}-4,78 \mathrm{~cm}$ & 67.5 & 483 & 2830.5 & 0 & 0 & 135 & 483 & 2830.5 & 0 & 0 & 3448.5 \\
\hline $14 \mathrm{R}-3,103 \mathrm{~cm}$ & 55 & 255 & 787.5 & 0 & 0 & 110 & 1020 & 787.5 & 0 & 0 & 1917.5 \\
\hline $15 \mathrm{R}-1,55 \mathrm{~cm}$ & 60 & 234 & 722 & 0 & 0 & 120 & 936 & 722 & 0 & 0 & 1778 \\
\hline $15 \mathrm{R}-3,72 \mathrm{~cm}$ & 45 & 280 & 1386.5 & 0 & 0 & 90 & 1120 & 1386.5 & 0 & 0 & 2596.5 \\
\hline $16 \mathrm{R}-2,37 \mathrm{~cm}$ & 71.5 & 312 & 1563.5 & 0 & 0 & 143 & 1248 & 1563.5 & 0 & 0 & 2954.5 \\
\hline
\end{tabular}


Table 1 (continued).

\begin{tabular}{|c|c|c|c|c|c|c|c|c|}
\hline \multicolumn{5}{|c|}{ Clay mineral percentage } & \multicolumn{2}{|c|}{ Peak height $(\mathrm{cm})$} & \multicolumn{2}{|c|}{$\begin{array}{l}\text { Clay mineral } \\
\text { percentage }\end{array}$} \\
\hline $\begin{array}{l}\text { Kaolinite } \\
+ \text { chlorite }\end{array}$ & Illite & Smectite & Palygorskite & Sepiolite & Kaolinite & Chlorite & Kaolinite & Chlorite \\
\hline 29 & 22 & 49 & 0 & 0 & 110 & 89 & 16 & 13 \\
\hline 42 & 49 & 9 & 0 & 0 & 124 & 121 & 22 & 20 \\
\hline 36 & 49 & 15 & 0 & 0 & 76 & 68 & 19 & 17 \\
\hline 41 & 53 & 6 & 0 & 0 & 85 & 69 & 23 & 18 \\
\hline 48 & 43 & 9 & 0 & 0 & 82 & 50 & 30 & 18 \\
\hline 36 & 54 & 10 & 0 & 0 & 85 & 75 & 19 & 17 \\
\hline 23 & 45 & 33 & 0 & 0 & 36 & 29 & 13 & 10 \\
\hline 13 & 40 & 47 & 0 & 0 & 25 & 17 & 8 & 5 \\
\hline 21 & 49 & 30 & 0 & 0 & 47 & 39 & 12 & 9 \\
\hline 21 & 36 & 43 & 0 & 0 & 27 & 18 & 13 & 8 \\
\hline 38 & 14 & 48 & 0 & 0 & 84 & 50 & 24 & 14 \\
\hline 37 & 41 & 22 & 0 & 0 & 127 & 75 & 23 & 14 \\
\hline 27 & 36 & 37 & 0 & 0 & 55 & 67 & 15 & 12 \\
\hline 23 & 50 & 27 & 0 & 0 & $?$ & 5 & $?$ & 23 \\
\hline 17 & 47 & 36 & 0 & 0 & $?$ & 5 & $?$ & 17 \\
\hline 19 & 45 & 36 & 0 & 0 & ? & 7 & $?$ & 19 \\
\hline 22 & 43 & 35 & 0 & 0 & ? & 3 & ? & 22 \\
\hline 18 & 52 & 30 & 0 & 0 & $?$ & 6 & $?$ & 18 \\
\hline 27 & 50 & 23 & 0 & 0 & 21 & 19 & 14 & 13 \\
\hline 20 & 32 & 33 & 15 & 0 & 62 & 31 & 13 & 7 \\
\hline 37 & 28 & 20 & 15 & 0 & 93 & 47 & 24 & 13 \\
\hline 7 & 22 & 19 & 52 & 0 & 35 & 25 & 4 & 3 \\
\hline 17 & 39 & 16 & 28 & 0 & 83 & 53 & 11 & 6 \\
\hline 22 & 48 & 30 & 0 & 0 & 80 & 56 & 13 & 9 \\
\hline 9 & 78 & 13 & 0 & 0 & $?$ & $?$ & $?$ & $?$ \\
\hline 5 & 33 & 62 & 0 & 0 & ? & ? & $?$ & $?$ \\
\hline 9 & 21 & 44 & 26 & 0 & 35 & 25 & 5 & 4 \\
\hline 15 & 22 & 35 & 28 & 0 & 57 & 27 & 10 & 5 \\
\hline 7 & 20 & 24 & 49 & 0 & 67 & 41 & 4 & 3 \\
\hline 19 & 21 & 24 & 36 & 0 & 65 & 44 & 11 & 8 \\
\hline 9 & 19 & 18 & 54 & 0 & 59 & 31 & 6 & 3 \\
\hline 15 & 23 & 26 & 36 & 0 & 103 & 72 & 9 & 6 \\
\hline$<1$ & 20 & 55 & 25 & 0 & $?$ & ? & $?$ & $?$ \\
\hline 3 & 39 & 58 & 0 & 0 & $?$ & $?$ & $?$ & $?$ \\
\hline 9 & 51 & 40 & 0 & 0 & ? & $?$ & $?$ & $?$ \\
\hline 13 & 39 & 48 & 0 & 0 & 18 & 20 & 6 & 7 \\
\hline 24 & 35 & 41 & 0 & 0 & 71 & 69 & 12 & 12 \\
\hline 15 & 39 & 46 & 0 & 0 & 30 & 35 & 7 & 8 \\
\hline 19 & 36 & 45 & 0 & 0 & 61 & 60 & 10 & 9 \\
\hline 30 & 41 & 29 & 0 & 0 & 98 & 93 & 15 & 15 \\
\hline 18 & 34 & 48 & 0 & 0 & 124 & 87 & 11 & 7 \\
\hline 21 & 41 & 38 & 0 & 0 & 110 & 77 & 12 & 9 \\
\hline 30 & 30 & 40 & 0 & 0 & 100 & 69 & 18 & 12 \\
\hline 29 & 34 & 37 & 0 & 0 & 63 & 46 & 17 & 12 \\
\hline 15 & 17 & 68 & 0 & 0 & 8 & 0 & 15 & 0 \\
\hline 22 & 23 & 55 & 0 & 0 & 11 & 0 & 22 & 0 \\
\hline 21 & 24 & 55 & 0 & 0 & 9 & 0 & 21 & 0 \\
\hline 29 & 26 & 45 & 0 & 0 & 9 & 0 & 29 & 0 \\
\hline 22 & 22 & 56 & 0 & 0 & 12 & 0 & 22 & 0 \\
\hline 0 & 20 & 41 & 39 & 0 & 0 & 0 & 0 & 0 \\
\hline 3 & 29 & 47 & 21 & 0 & 0 & 0 & 0 & 0 \\
\hline 5 & 37 & 47 & 11 & 0 & 0 & 0 & 0 & 0 \\
\hline 9 & 61 & 30 & 0 & 0 & 0 & 0 & 0 & 0 \\
\hline 4 & 35 & 61 & 0 & 0 & 0 & 0 & 0 & 0 \\
\hline 3 & 34 & 63 & 0 & 0 & 0 & 0 & 0 & 0 \\
\hline 4 & 37 & 59 & 0 & 0 & 0 & 0 & 0 & 0 \\
\hline 3 & 35 & 62 & 0 & 0 & 0 & 0 & 0 & 0 \\
\hline 4 & 42 & 54 & 0 & 0 & 0 & 0 & 0 & 0 \\
\hline 6 & 35 & 59 & 0 & 0 & 0 & 0 & 0 & 0 \\
\hline 3 & 24 & 73 & 0 & 0 & 0 & 0 & 0 & 0 \\
\hline 4 & 47 & 49 & 0 & 0 & 0 & 0 & 0 & 0 \\
\hline 2 & 28 & 70 & 0 & 0 & 0 & 0 & 0 & 0 \\
\hline 3 & 13 & 84 & 0 & 0 & 0 & 0 & 0 & 0 \\
\hline 2 & 27 & 71 & 0 & 0 & 0 & 0 & 0 & 0 \\
\hline 6 & 52 & 42 & 0 & 0 & 0 & 0 & 0 & 0 \\
\hline 10 & 34 & 56 & 0 & 0 & 0 & 0 & 0 & 0 \\
\hline 6 & 44 & 50 & 0 & 0 & 0 & 0 & 0 & 0 \\
\hline 5 & 41 & 54 & 0 & 0 & 0 & 0 & 0 & 0 \\
\hline 0 & 46 & 54 & 0 & 0 & 0 & 0 & 0 & 0 \\
\hline 5 & 53 & 42 & 0 & 0 & 0 & 0 & 0 & 0 \\
\hline 11 & 60 & 29 & 0 & 0 & 0 & 0 & 0 & 0 \\
\hline 11 & 53 & 36 & 0 & 0 & 0 & 0 & 0 & 0 \\
\hline 3 & 53 & 44 & 0 & 0 & 0 & 0 & 0 & 0 \\
\hline 5 & 40 & 55 & 0 & 0 & 0 & 0 & 0 & 0 \\
\hline 4 & 14 & 82 & 0 & 0 & 0 & 0 & 0 & 0 \\
\hline 6 & 53 & 41 & 0 & 0 & 0 & 0 & 0 & 0 \\
\hline 7 & 53 & 40 & 0 & 0 & 0 & 0 & 0 & 0 \\
\hline 4 & 43 & 53 & 0 & 0 & 0 & 0 & 0 & 0 \\
\hline 5 & 42 & 53 & 0 & 0 & 0 & 0 & 0 & 0 \\
\hline
\end{tabular}


Table 2. Characteristic weaks and correction factors for clay minerals detected in samples from Leg 103 .

\begin{tabular}{lcc}
\hline \multicolumn{1}{c}{$\begin{array}{c}\text { Main peak(s) used } \\
\text { Mineral }\end{array}$} & $\begin{array}{l}\left({ }^{\circ} 2 \theta\right) \\
\text { Kor identification } \\
\text { Kaolinite }\end{array}$ & Correction factor \\
Illite & $12.4 / 24.88$ & 2 \\
Chlorite & 8.8 & 4 \\
a Smectite, & $6.2 / 12.4 / 25.15$ & 2 \\
$\quad$ glycolated & $5.0-5.5$ & 1 \\
Palygorskite & 8.5 & 5 \\
Sepiolite & 7.3 & 5 \\
\hline
\end{tabular}

a Smectite is used here as a blanket term for all smectites and illite-smectite interlayers that expand to give a $17 \AA$, $5.0^{\circ}-5.5^{\circ} 2 \theta$ peak on glycolation.

Lithologic Unit VI, termed the "problematic interval" by the shipboard sedimentologists, includes a wide variety of very poorly recovered sedimentary, igneous, and metamorphic rocks (see "Site 639" chapter; Boillot, Winterer, et al., 1987). No samples were taken for clay analyses.

\section{Site 640}

Drilling at Site 640 was on a buried north-trending ridge near the western edge of the Galicia margin that brings "acoustic basement" (actually a zone of incoherent reflectors) close to the seafloor (Fig. 1). Only a few cores were drilled into this acoustically incoherent unit (Cores 103-640A-5R through 103-640A-9R), to test the hypothesis that the deeper $\mathrm{S}$ reflector within "acoustic basement" represents the boundary between ductile and brittle crust, where listric faults merge at depth (Montadert et al., $1979 \mathrm{~b}$; Chenet et al., 1982). The section from 0 to $145 \mathrm{mbsf}$ was washed without coring. The recovered sediments below that depth are divided into three lithologic units (see "Site 640" chapter; Boillot, Winterer, et al., 1987). Clay samples were analyzed from all three units. Figure 5 shows the downhole trends in clay mineralogy at this site.

Lithologic Unit I (145-157 mbsf) consists of pelagic brown and yellowish brown clay, interpreted to have accumulated below the CCD. The clay is faintly mottled and with diffuse banding on a scale of 1-10 cm. Shipboard smear slide analysis shows the sediment to be composed principally of recrystallized clay minerals, with trace amounts of quartz silt and carbonate rhombs. Based on ichthyoliths, this unit is of Paleocene age. The clay component of the samples analyzed from this unit averages about $20 \%$ illite, $30 \%$ smectite, and $35 \%$ palygorskite, with smaller amounts of kaolinite and chlorite (totaling about 10\%-15\%).

Lithologic Unit II (157-165 mbsf) consists of Albian-Aptian olive-gray clay and white and olive-yellow clayey ooze and is interpreted as a pelagic deposit. The few clay samples analyzed from this unit consist dominantly of smectite (about 55\%) and illite (about $40 \%$ ), with only a few percent kaolinite and chlorite.

Lithologic Unit III (165-232 mbsf) consists of Barremian interbedded dark gray calcareous claystone and marlstone and white clayey chalk (Subunit IIIA), overlying Hauterivian sandstone, limestone, and marlstone (Subunit IIIB). This unit is interpreted as being a sequence of interbedded distal terrigenous turbidites and pelagic and hemipelagic sediments. Only calcareous clay and marl were sampled from this unit for clay mineralogic analyses. The samples consist of an average of $10 \%$ kaolinite, $10 \%$ chlorite, $40 \%$ illite, and $40 \%$ smectite.

\section{Site 641}

Site 641 was situated upslope and east of Site 638 (Fig. 1), so that drilling would supplement data from Site 638 by coring the upper part of the syn-rift and the lower part of the post-rift sections. A major objective at Site 641 was to date the break-up unconformity on the Galicia margin. Cores were collected from two holes at this site: Hole $641 \mathrm{~A}$, cored from 0-63.6 mbsf, and Hole $641 \mathrm{C}$, cored from 150.9-305.2 mbsf. The interval from 63.6 to $150.9 \mathrm{mbsf}$ was not cored. The recovered sediments are divided into six lithologic units. Clay profiles for samples analyzed from this site are given in Figures 6 (Hole 641A) and 7 (Hole 641C).

Lithologic Unit I (0-53.6 mbsf), cored only in Hole 641A, consists principally of uniform, structureless, brown and dark grayish brown Upper Cretaceous (Turonian-Maestrichtian) clay that becomes redder downhole. It is interpreted as being a pelagic clay, accumulating slowly in an oxygenated environment below the CCD. The uppermost $15 \mathrm{~m}$ of this unit also includes slumped and highly disturbed intervals of brown clay, marl, and calcareous ooze of early Pleistocene age (Moullade et al., this volume). The clay mineralogy of brown clay samples from this unit averages $20 \%$ kaolinite + chlorite, $30 \%$ illite, and $50 \%$ smectite. Smectite increases downhole at the expense of illite; kaolinite is not detected below Core 103-641A-4X. None of the samples contain detectable quantities of palygorskite.

Below lithologic Unit I, Hole 641A is divided into lithologic Unit II (53.6-53.9 mbsf) and lithologic Subunit IIIA (53.9$>63.6 \mathrm{mbsf}$ ). Lithologic Unit II consists of black zeolitic clay with approximately $10 \%$ organic matter (Dunham et al., this volume). Lithologic Subunit IIIA consists of upper Albian-Cenomanian greenish gray calcareous clay and marl. No samples were taken from either of these units for clay analyses.

Sediments recovered from Hole $641 \mathrm{C}$ are divided into lithologic Subunit IIIB and Units IV through VI (Fig. 7). Subunit IIIB ( $<150.9-202.6 \mathrm{mbsf})$ consists of alternations of lower middle Albian black, dark green, organic-rich laminated claystone, with minor marlstone and very fine-grained sandstone and siltstone laminae. The subunit is characterized by its abundant black claystone layers. Lithologic Unit IV (202.6-218.4 mbsf) consists of upper Aptian greenish gray marlstone and calcareous claystone, with several interbeds of limestone conglomerate and coarse-grained calcarenite. The coarse-grained beds are probably turbidites. Lithologic Units V (218.4-250.6 mbsf) and VI (250.6-305.2 mbsf) consist of alternating intervals of thin-bedded, upper Barremian-lower Aptian greenish gray calcareous claystone and marlstone microturbidites, bioturbated and faintly laminated gray marlstone, and bioturbated, massive clayey limestone. Unit VI includes minor debris flows and sand turbidites, and is distinguished from Unit V by being richer in carbonate overall. Detailed descriptions of these sedimentary units are given in the "Site 641" chapter (Boillot, Winterer, et al., 1987).

All clay samples analyzed from Hole $641 \mathrm{C}$ contain only minor amounts of kaolinite and chlorite $(0 \%-11 \%)$ (Fig. 7). Illite percentages range from $13 \%$ to $61 \%$, averaging $40 \%$; smectite ranges from $29 \%$ to $84 \%$, averaging $55 \%$. In general, the more carbonate-rich greenish gray calcareous claystone and marlstone samples contain greater amounts of illite and lesser amounts of smectite than do the darker gray or black claystone samples. Only samples from the uppermost portion of Subunit IIIB (Cores 103-641C-1R and 103-641C-2R) contain detectable amounts of palygorskite.

\section{DISCUSSION AND CONCLUSIONS}

\section{Neogene Sediments}

Neogene nannofossil marl, clayey nannofossil ooze, and calcareous clay were recovered at Hole 637A (lithologic Units I and II; 0-185 mbsf), Hole 638B (lithologic Unit I; 0-183.6 mbsf), and Hole 639A (lithologic Unit I). These calcareous sediments are interbedded with clay and silty clay terrigenous turbidites in 


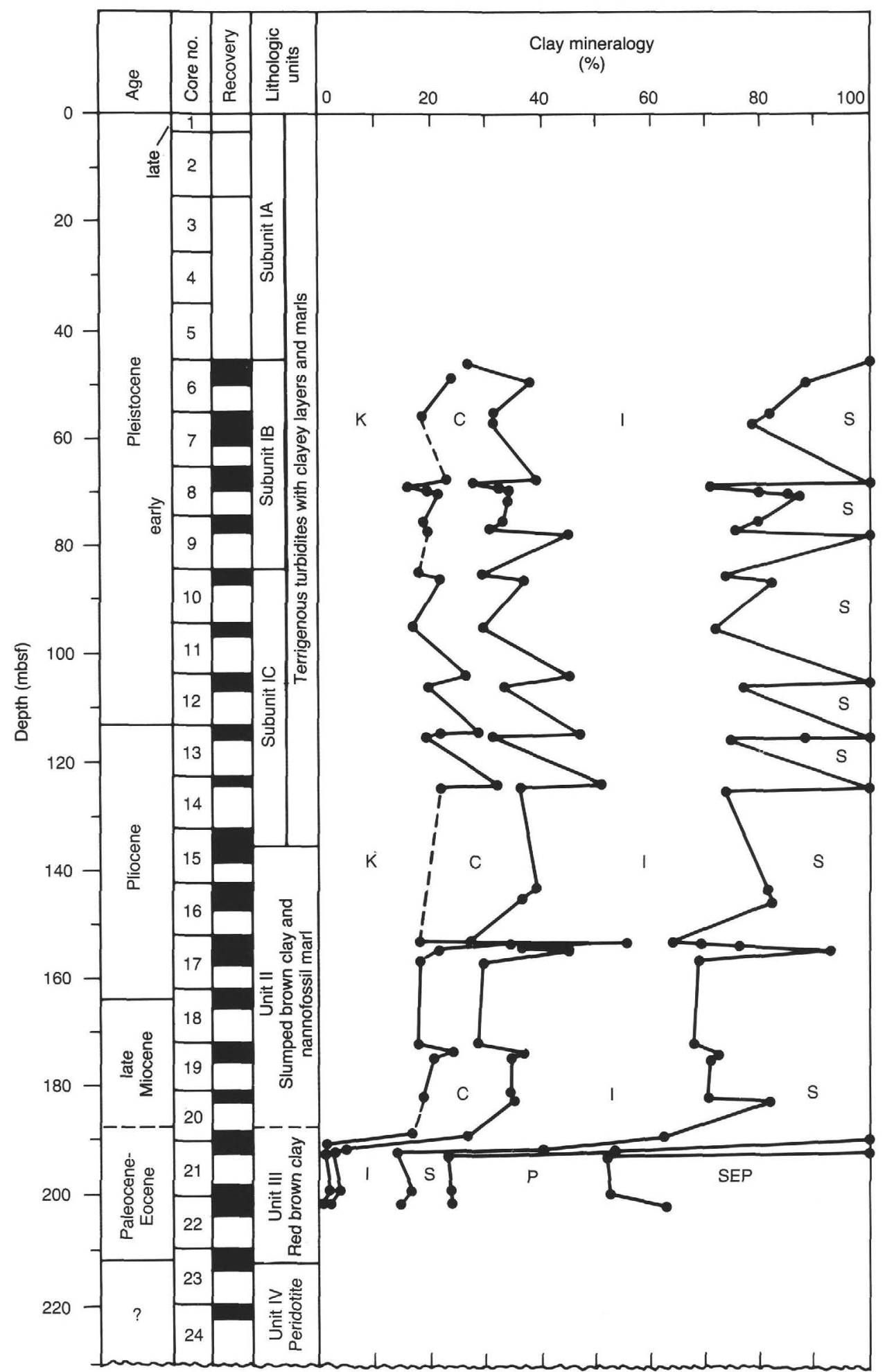

Figure 2. Age, core number and recovery (in black), lithologic units, and clay mineralogy of Site 637 (Hole 637A) samples. $\mathrm{K}=$ kaolinite; $\mathrm{C}=$ chlorite; $\mathrm{I}=$ illite; $\mathrm{S}=$ smectite, $\mathrm{P}=$ palygorskite; $\mathrm{SEP}=$ sepiolite. Age information from Moullade et al. (this volume).

lithologic Unit I of Hole 637A. The calcareous sediments primarily appear to be pelagic carbonates deposited above the CCD, though the more clay-rich intervals may represent increased influx of terrigenous material during Pliocene-Pleistocene glaciations, perhaps as distal turbidity flows or as nepheloid layers. The clay mineralogy of the calcareous sediments is similar at all sites: $10 \%-20 \%$ kaolinite, $10 \%-15 \%$ chlorite, $40 \%-50 \%$ illite, and $25 \%-35 \%$ smectite. Smectite percentages are related to the carbonate content of the sediments: the clayey nannofossil ooze and marl samples contain greater percentages of smectite than do the calcareous clays or the clay and silty clay turbidites. This suggests that, relative to the clay component of the background 


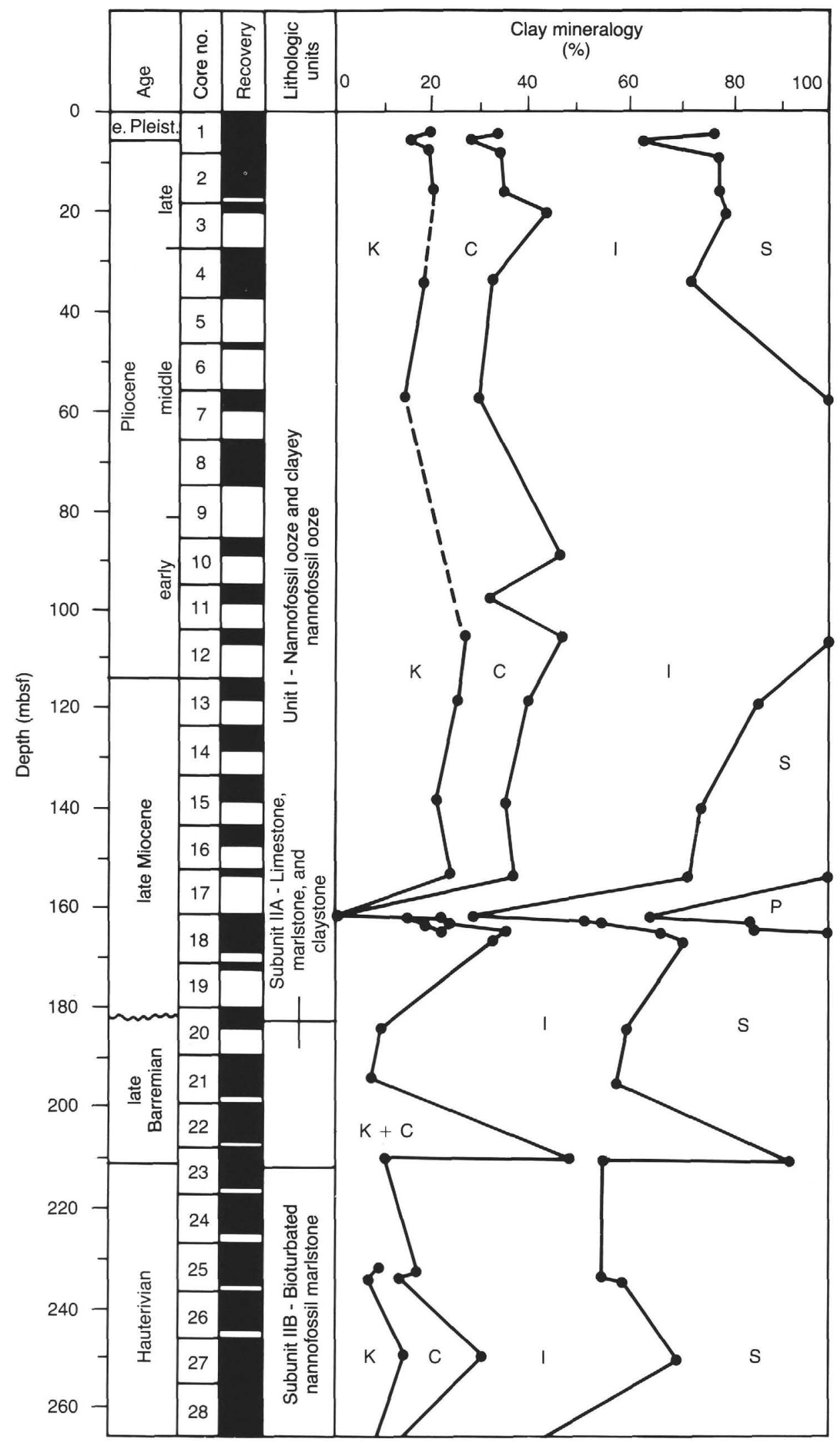

Figure 3. Age, core number and recovery (in black), lithologic units, and clay mineralogy of Site 638 (Holes 638B and 638C) samples. $\mathrm{K}=$ kaolinite; $\mathrm{C}=$ chlorite; $\mathrm{I}=$ illite; $\mathrm{S}=$ smectite; $\mathrm{P}=$ palygorskite. Age information from Moullade et al. (this volume). Core numbers from Hole $638 \mathrm{C}$ are prefaced by a "C." 


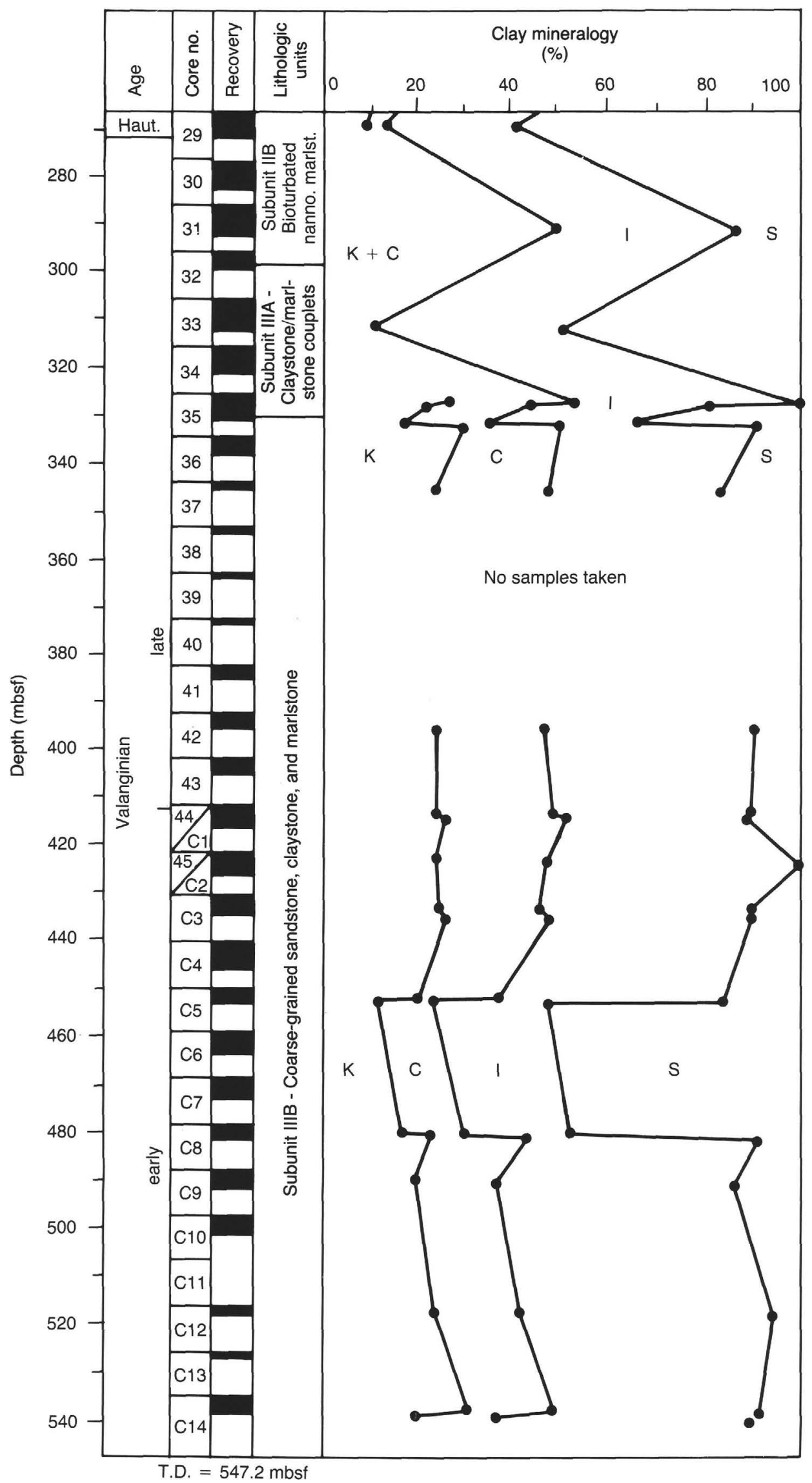

Figure 3 (continued). 


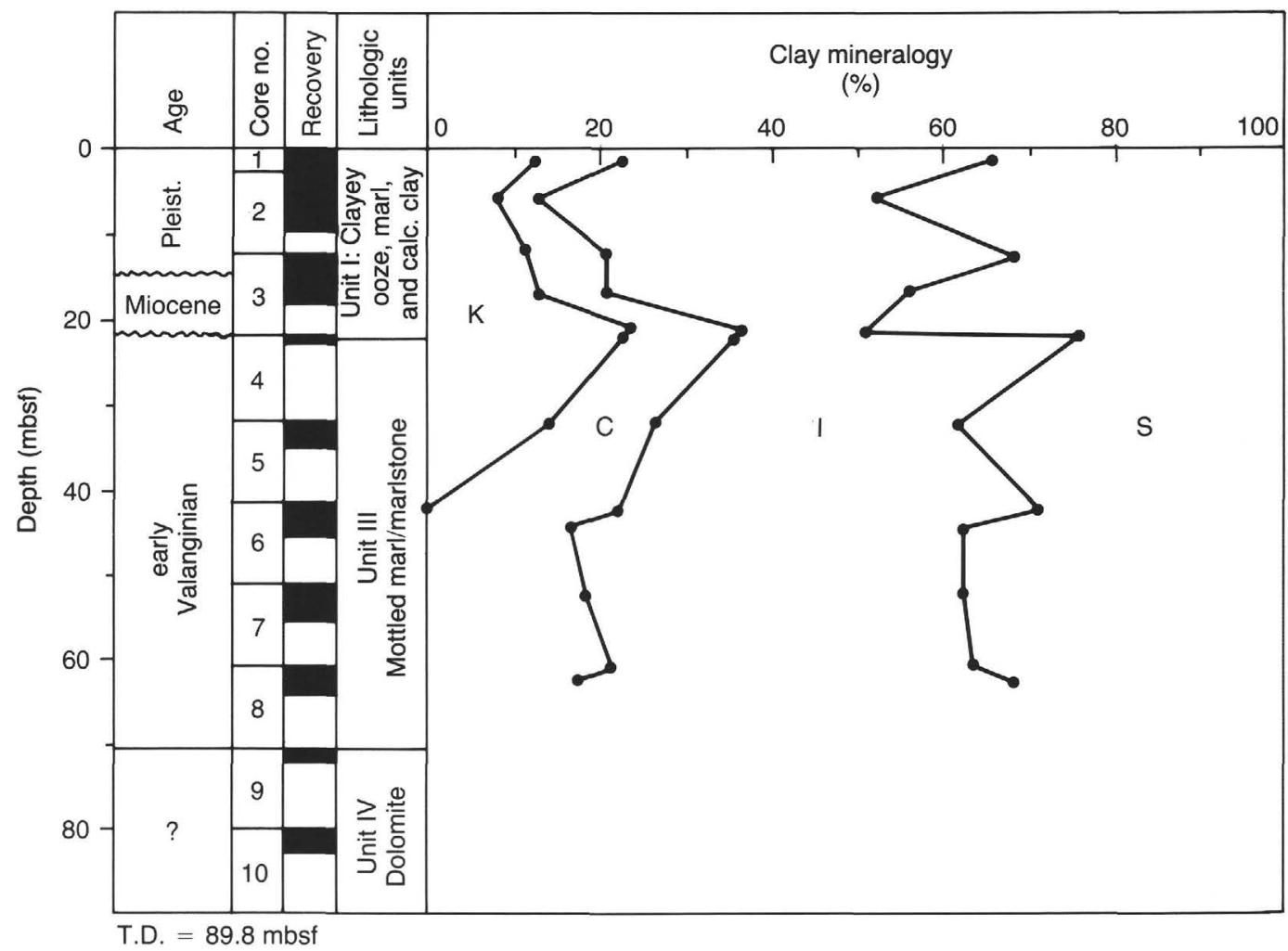

Figure 4. Age, core number and recovery (in black), lithologic units, and clay mineralogy of Site 639 (Hole 639A) samples. $\mathrm{K}=$ kaolinite; $\mathrm{C}=$ chlorite; $\mathrm{I}=$ illite; $\mathrm{S}=$ smectite. Age information from Moullade et al. (this volume).

pelagic sediment, the sources of fine-grained terrigenous sediment to the region contained little smectite during the Neogene.

These results are in general agreement with those of Chamley et al. (1979), who analyzed the clay mineralogy of Tertiary and Cretaceous sediments recovered at Site 398. Because the two studies used different peak analysis methods and different correction factors, direct comparison of the data sets is not possible. However, in their "Zone 1," which includes sediments of Oligocene-Pleistocene age, Chamley et al. (1979) documented an increasing abundance of illite and chlorite upsection. They attributed this to be the result of a generally cooling climate during that time period. This same abundance of illite and chlorite, relative to that in the underlying Cretaceous sediments, is seen in the Leg 103 samples. This is especially well documented in the silty clay and clay turbidites derived from the adjacent continent.

Initial shipboard interpretation of the depositional environment of the late Miocene-Pliocene age slumped brown clays and nannofossil marls at Site 637 (lithologic Unit II; 135-185 mbsf) suggested that the unit included weathering products from the underlying peridotite basement, slumped off the basement outcrop exposed a short distance to the west of the site (Fig. 1). The underlying brown clays of lithologic Unit III contain significant amounts of sepiolite, interpreted as being derived from alteration of the peridotite basement (see the following discussion). However, sepiolite is not found in any of the lithologic Unit II samples analyzed. This lack of sepiolite in lithologic Unit II suggests that the slumps in this unit do not incorporate significant weathering or alteration products from the basement ridge. Instead, the source of the slumped material in lithologic Unit II probably consists primarily of pelagic material deposited on top of the ridge.

\section{Brown Clays}

Brown, reddish brown, and grayish brown clays were recovered at all five Leg 103 sites: Hole 637A (lithologic Unit III; 185-212 mbsf), Hole 638B (as redeposited material within lithologic Unit I), Hole 639C (lithologic Unit II), Hole 640A (lithologic Unit I; 145-157 mbsf), and Hole 641A (lithologic Unit I; $0-53.6 \mathrm{mbsf}$ ).

Based on ichthyoliths, the brown clays at Holes 637A, 639C, and $640 \mathrm{~A}$ are of Paleocene-Eocene age. The brown clay at Hole $638 \mathrm{~B}$ could also be of similar age, as it was redeposited during the Neogene but contains reworked microfossils. All samples analyzed from the brown clays of these four holes contain significant percentages of palygorskite $(15 \%-46 \%)$. Both illite and smectite occur in percentages ranging from $10 \%$ to $30 \%$, whereas kaolinite and chlorite are present in smaller amounts (usually less than 10\%). These similarities in age, lithology, and clay mineralogy suggest a common source for all these clay units.

The brown clay of lithologic Unit III at Hole 637A differs from those in Holes $638 \mathrm{~B}, 639 \mathrm{C}$, and $640 \mathrm{~A}$ in that it also contains significant amounts $(36 \%-48 \%)$ of sepiolite. This likely formed in association with the serpentinization and alteration of the immediately underlying peridotite basement ridge. (See Boillot et al. (1980) for a discussion of the alteration history of the basement ridge as determined from dredge haul samples collected on an exposed section of the ridge immediately west of Site 637.)

The brown clay recovered in Hole 641A appears to be different from that recovered at the other four sites. Though it is lithologically similar to the other brown clays, it is of Late Cretaceous age and does not contain detectable amounts of palygorskite. Clearly, the source of clay material to the Galicia margin 


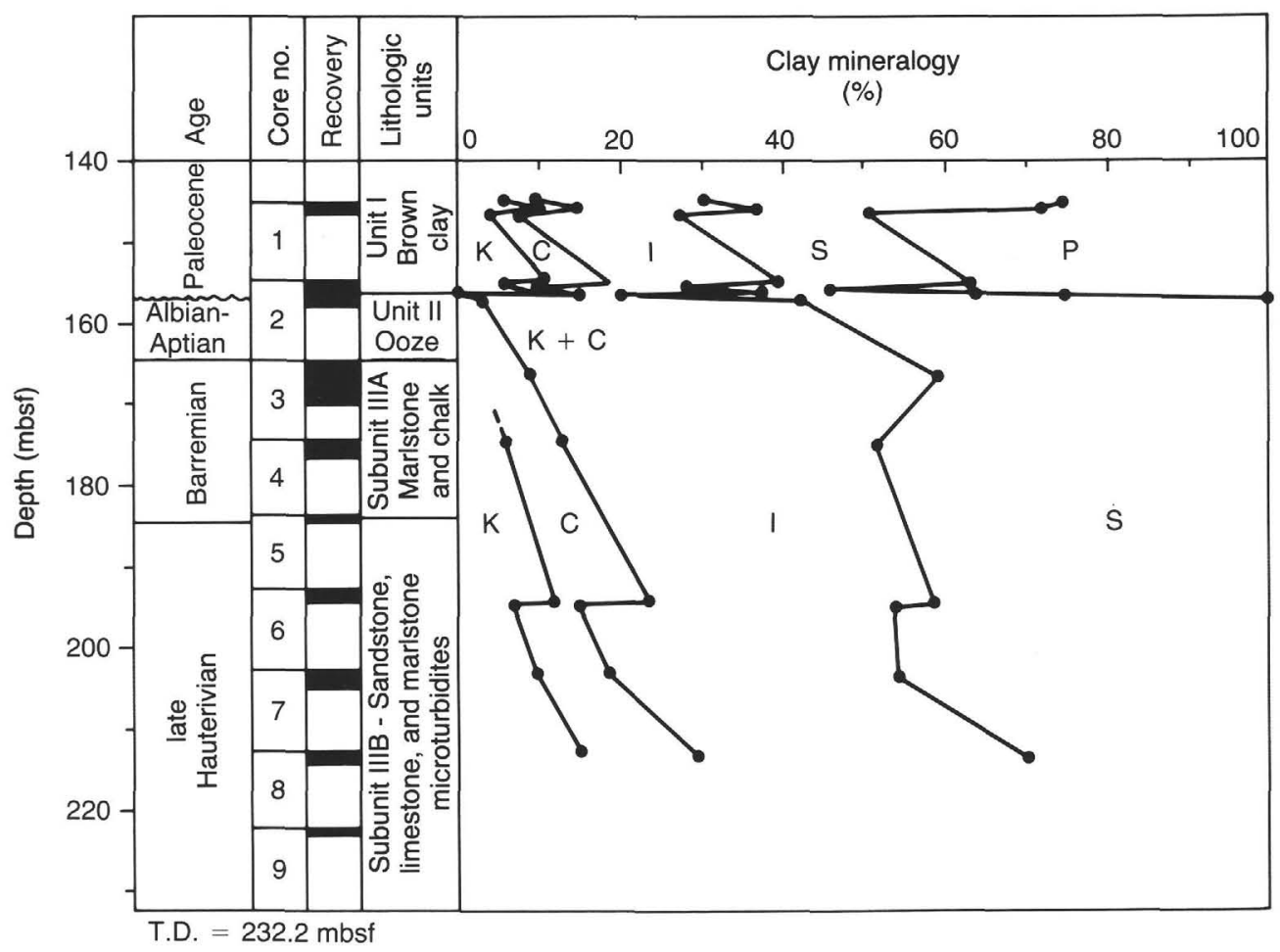

Figure 5. Age, core number and recovery (in black), lithologic units, and clay mineralogy of Site 640 (Hole 640A) samples. $\mathrm{K}=$ kaolinite; $\mathrm{C}=$ chlorite; $\mathrm{I}=$ illite; $\mathrm{S}=$ smectite, $\mathrm{P}=$ palygorskite. Age information from Moullade et al. (this volume).

during the Late Cretaceous contained relatively less palygorskite and relatively more smectite than during Paleocene-Eocene time (see Chamley et al. (1979) for a similar interpretation).

\section{Cretaceous Sediments}

The Cretaceous post- and syn-rift section drilled during Leg 103 was poorly sampled for clay analyses. Accordingly, only generalized conclusions can be reached about the source of clays in these sediments or the paleoenvironment. As is the case with the overlying Cenozoic section, the more calcareous, pelagic portions of the Cretaceous section contain greater amounts of smectite and lesser amounts of kaolinite and chlorite than the more terrigenous, clay-rich sediments. Apparently, the source of terrigenous fine-grained sediment throughout the syn- and post-rift period contained less smectite than that present in the pelagic material deposited between turbidity events. Also, the overall abundance of smectite in the Upper Cretaceous sediments is greater than that in the Tertiary sediments. As discussed by Chamley et al. (1979), this supports the interpretation of a warmer climate during Cretaceous time.

\section{ACKNOWLEDGMENTS}

The authors wish to thank Bettina Domeyer, Mark Dobday, and Jeri Wackler, who helped in various stages of the clay sample preparation, data collection, and profile analysis. Partial support for this study was provided by the United States Science Advisory Committee (USSAC). This paper benefited from reviews provided by J. Schoonmaker, S. O'Connell, and G. Boillot.

\section{REFERENCES}

Biscaye, P. E., 1965. Mineralogy and sedimentation of Recent deep-sea clay in the Atlantic Ocean and adjacent seas and oceans. Geol. Soc. Am. Bull., 76:803-832.

Boillot, G., Auxiètre, J. L., Dunand, J. P., Dupeuble, P. A., and Mauffret, A., 1979. The northwestern Iberian margin: a Cretaceous pas- sive margin deformed during Eocene. In Talwani, M., Hay, W., and Ryan, W.B.F. (Eds.), Deep Drilling Results in the Atlantic Ocean: Continental Margins and Paleoenvironment: Am. Geophys. Union, Maurice Ewing Ser., 3:138-153.

Boillot, G., Grimaud, S., Mauffret, A., Mougenot, D., Kornprobst, J., Mergoil-Daniel, J., and Torrent, G., 1980. Ocean-continent boundary off the Iberian margin: a serpentinite diapir west of the Galicia Bank. Earth Planet. Sci. Lett., 48:23-34.

Boillot, G., Winterer, E. L., et al., 1987. Proc. ODP, Init. Repts., 103: College Station, TX (Ocean Drilling Program).

Chamley, H., Debrabant, P., Foulon, J., Giroud d'Argoud, G., Latouche, C., Maillet, N., Maillot, H., and Sommer, F., 1979. Mineralogy and geochemistry of Cretaceous and Cenozoic Atlantic sediments off the Iberian Peninsula (Site 398, DSDP Leg 47B). In Sibuet, J.-C., Ryan, W.B.F., et al., Init. Repts. DSDP, 47, Pt. 2: Washington (U.S. Govt. Printing Office), 429-444.

Chenet, P., Montadert, L., Gairaud, H., and Roberts, D. G., 1982. Extension ratio measurements on the Galicia, Portugal, and northern Biscay continental margins: implications for evolutionary models of passive continental margins. In Watkins, J. S., and Drake, C. L. (Eds.), Studies in Continental Margin Geology: AAPG Mem., 34: 703-715.

Groupe Galice, 1979. The continental margin off Galicia and Portugal: acoustical stratigraphy, dredge stratigraphy and structural evolution. In Sibuet, J.-C., Ryan, W.B.F., et al., Init. Repts. DSDP, 47, Pt. 2: Washington (U.S. Govt. Printing Office), 633-662.

Hein, J. R., Scholl, D. W., and Gutmacher, C. E., 1976. Neogene clay minerals of the far NW Pacific and southern Bering Sea-sedimentation and diagenesis. Proc. Int. Clay Conf. 1975, 71-80.

Milliman, J. D., and Bornhold, B. D., 1973. Discussion: peak height versus peak intensity analysis of X-ray diffraction data. Sedimentology, 20:445-448.

Montadert, L., de Charpal, O., Robert, D., Guennoc, P., and Sibuet, J.-C., 1979a. Northeast Atlantic passive continental margin: rifting and subsidence processes. In Talwani, M., Hay, W., Ryan, W.B.F. (Eds.), Deep Drilling Results in the Atlantic Ocean: Continental Margins and Paleoenvironment: Am. Geophys. Union, Maurice Ewing Ser., 3:154-186. 
Montadert, L., Roberts, D. G., de Charpal, O., and Guennoc, P., 1979b. Rifting and subsidence of the northern continental margin of the Bay of Biscay. In Montadert, L., and Roberts, D. G., et al., Init. Repts. DSDP, 48: Washington (U.S. Govt. Printing Office), 10251060.

Montadert, L., Winnock, E., Delteil, J. R., and Grau, G., 1974. Continental margins of Galicia-Portugal and Bay of Biscay. In Burk, C. A., and Drake, C. L. (Eds.), The Geology of Continental Margins: Berlin (Springer-Verlag), 323-342.
Sibuet, J.-C., Ryan, W.B.F., et al., 1979. Init. Repts. DSDP, 47, Pt. 2: Washington (U.S. Govt. Printing Office).

Date of initial receipt: 28 April 1987

Date of acceptance: 2 February 1988

Ms 103B-131

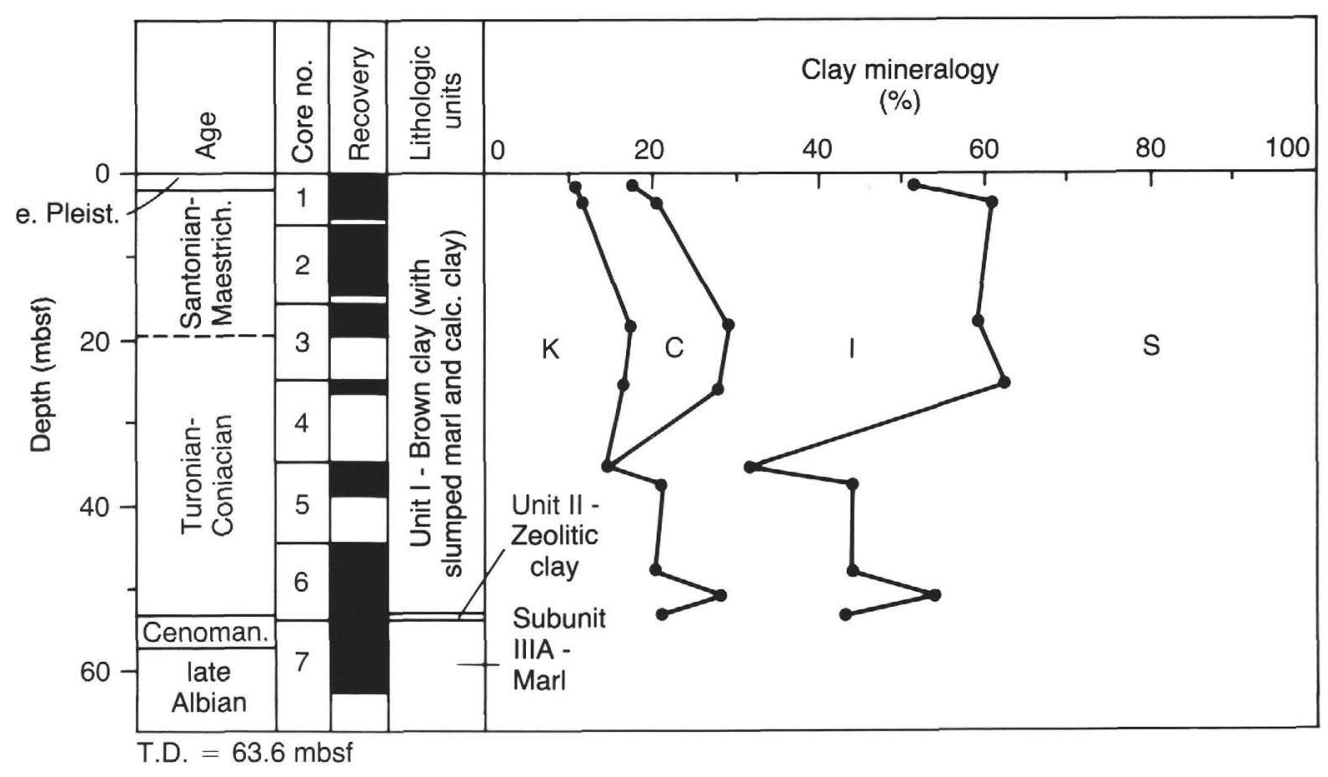

Figure 6. Age, core number and recovery (in black), lithologic units, and clay mineralogy of Hole 641A samples. $\mathrm{K}=$ kaolinite; $\mathrm{C}=$ chlorite; $\mathrm{I}=$ illite; $\mathrm{S}=$ smectite. Age information from Moullade et al. (this volume). 


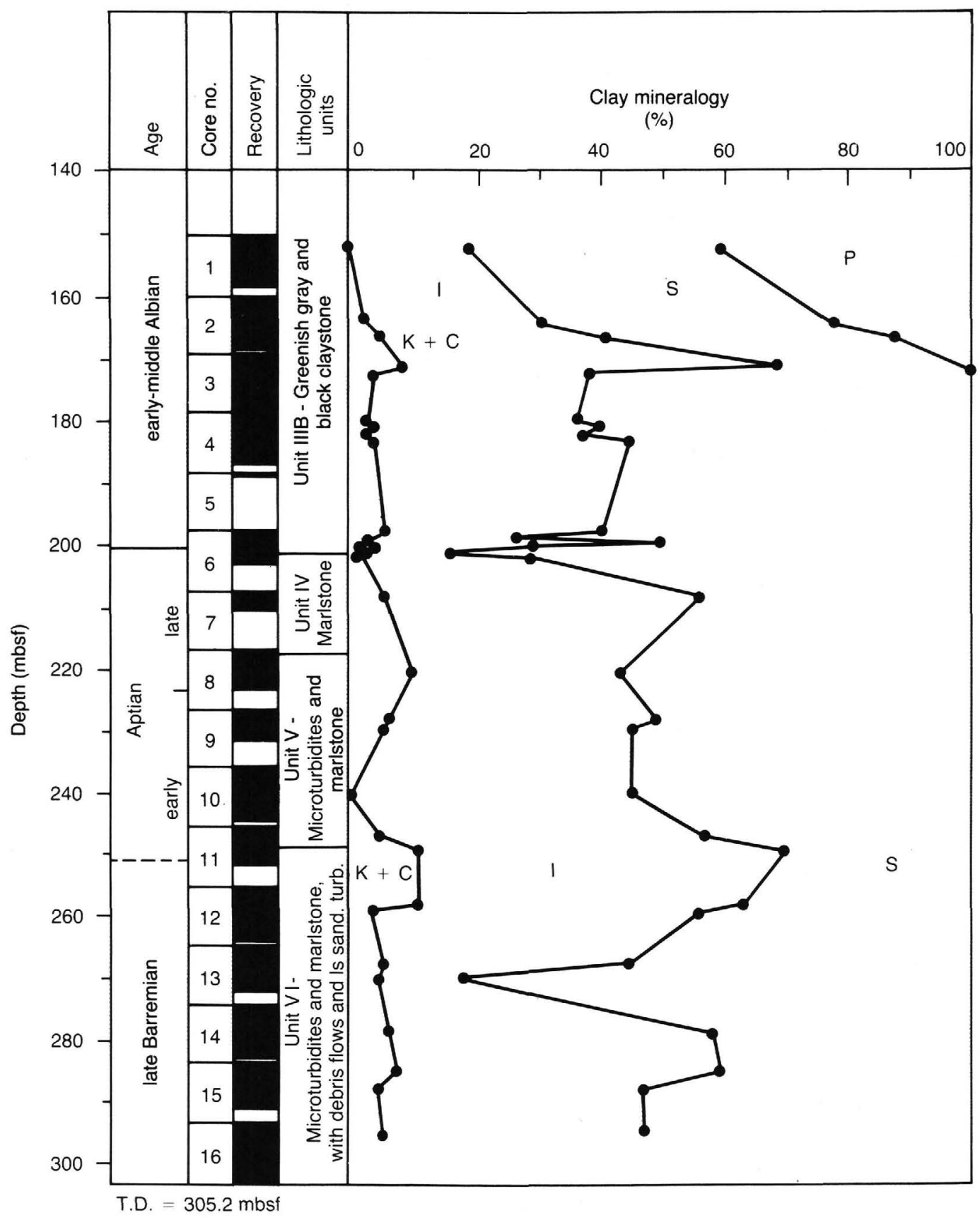

Figure 7. Age, core number and recovery (in black), lithologic units, and clay mineralogy of Hole 641C samples. K = kaolinite, $\mathrm{C}=$ chlorite, $\mathrm{I}=$ illite; $\mathrm{S}=$ smectite; $\mathrm{P}=$ palygorskite. Age information from Moullade et al. (this volume). 